\title{
TRATAMENTO CIRÚRGICO DA HIPERTENSÃO PORTAL ESQUISTOSSOMÓTICA NO HC/UFPE - ANÁLISE DE 131 CASOS
}

\section{SURGICAL TREATMENT OF THE SCHISTOSSOMOTIC PORTAL HYPERTENSION IN THE HC/UFPE - ANALYSIS OF 131 CASES}

\author{
Álvaro Antônio Bandeira Ferraz, TCBC-PE ${ }^{1}$ \\ Edmundo Pessoa de Almeida Lopes ${ }^{2}$ \\ Tércio Souto Bacelar TCBC-PE ${ }^{3}$ \\ Marcello Jorge de Castro Silveira, TCBC-PE ${ }^{3}$ \\ Luciana Medici Maranhão Silva ${ }^{4}$ \\ Edmundo Machado Ferraz,TCBC-PE ${ }^{5}$
}

\begin{abstract}
RESUMO: Objetivo: A hipertensão portal esquistossomótica com antecedente de hemorragia digestiva foi tratada com esplenectomia + ligadura da veia gástrica esquerda (LVGE) + desvascularização da grande curvatura do estômago + esclerose endoscópica pós-operatória. Quando da existência de varizes de fundo gástrico, realizamos a abertura do fundo gástrico e sutura obliterante destas varizes. O objetivo deste trabalho foi avaliar a recidiva de hemorragia digestiva, repercussões laboratoriais e mortalidade do tratamento cirúrgico/endoscópico. Métodos: Entre 1992 e 1998, foram operados no HC-UFPE 131 pacientes. O seguimento médio foi de 30 meses, em 111 pacientes, que foram solicitados a retornar ao ambulatório do HC-UFPE para a realização de controle clínico e laboratorial. Resultados: A recidiva hemorrágica foi de 14,4\% (16/111) e uma mortalidade de $5,4 \%(6 / 111)$. A recidiva de hemorragia digestiva alta foi exteriorizada através de hematemese em oito pacientes e oito por melena. Dos seis pacientes que foram a óbito, três apresentavam diagnóstico de linfoma, hepatocarcinoma e infarto agudo do miocárdio, respectivamente. Dois pacientes foram a óbito no pós-operatório imediato (sepse e coagulação intravascular disseminada). O sexto paciente foi a óbito por recidiva da hemorragia digestiva alta. Em nove pacientes, 13,2\%, foi diagnosticada trombose da veia porta. Os dados laboratoriais, hematológicos e de função hepática também foram analisados. Conclusões: Os autores concluíram que o tratamento cirúrgico da hipertensão portal esquistossomótica, através da esplenectomia + LVGE + desvascularização da grande curvatura do estômago + esclerose endoscópica pós-operatória determina resultados compatíveis com a literatura em relação à recidiva de sangramento, mas preserva a funcionalidade hepática.
\end{abstract}

Descritores: Esquistossomose hepato-esplênica; Esplenectomia; Trombose da veia porta.

\section{INTRODUÇÃO}

O tratamento cirúrgico da hipertensão portal esquistossomótica ainda é motivo de debate.
A hipertensão portal esquistossomótica apresenta, em sua fisiopatologia, um bloqueio pré-sinusoidal do fluxo portal $^{1}$. No entanto, algumas características deste tipo de hipertensão portal devem ser levadas em consideração na

1. Professor Adjunto do Departamento de Cirurgia da UFPE. Mestre e Doutor em Medicina pela UFPE. Especialização em Infecção em Cirurgia no Medical College of Wisconsin — EUA. Pós-Doutorado em Transplante de Fígado na Universidade de Miami — EUA.

2. Professor do Departamento de Medicina Clínica da UFPE.

3. Professor Adjunto do Departamento de Cirurgia da UFPE.

4. Aluna de Graduação do Curso de Medicina da UFPE.

5. Professor Titular de Cirurgia Abdominal e Bases da Técnica Cirúrgica da UFPE. Chefe do Serviço de Cirurgia Geral do Hospital das Clínicas da UFPE. Doutor e Livre-Docente pela UFPE. Fellow do American College of Surgeons (FACS) e do Surgical Infection Society.

Recebido em 1/12/99

Aceito para publicação em 25/7/2000

Trabalho apresentado no XXIII Congresso Brasileiro de Cirurgia — Rio de Janeiro — 1999. 
condução cirúrgica desta doença. Além do bloqueio présinusoidal, a ação do Shistossoma determina uma reação imunoreativa tanto do fígado quanto do baço. O tratamento clínico tem obtido sucesso na progressão do processo evolutivo, porém sem evidência de melhora das lesões hepáticas e hemodinâmicas já instaladas² ${ }^{2}$ A lesão hepática instalada preserva a arquitetura dos hepatócitos e a função hepática em níveis aceitáveis. Ou seja, apesar do bloqueio pré-sinusoidal, o fígado, na esquistossomose hepatoesplênica, mantém-se bem perfundido.

A história natural da variz de esôfago na fase hepatoesplênica da esquistossomose mansônica considera que um terço dos pacientes com esquistossomose hepatoesplênica não irá desenvolver hemorragia digestiva alta. Este risco é maior nos portadores de varizes de grosso calibre e menor nas varizes de fino calibre ${ }^{3}$. A mortalidade no primeiro sangramento destes pacientes foi de $11,7 \%{ }^{4}$. Analisando todos os casos de hemorragia digestiva alta em portadores de esquitossomose mansônica na forma hepatoesplênica, Silveira et al concluíram que o tratamento conservador (clínico/endoscópico) obtém bons resultados em mais de $90 \%$ dos $\operatorname{casos}^{4,5}$. Baseados nestes dados de história natural, consideramos que a hemorragia digestiva decorrente da esquitossomose mansônica apresenta um comportamento relativamente benigno, o qual, associado aos dados de fisiopatologia, fez com que fosse adotada uma conduta mais conservadora no tratamento cirúrgico destes pacientes 5 .

A indicação do tratamento cirúrgico, portanto, só é feita após um episódio de hemorragia digestiva e, na grande maioria das vezes, fora do quadro agudo de sangramento ${ }^{6}$.

Com base em uma experiência sólida e de resultados animadores, realizamos, desde a década de 50 com Kelner \& Wanderley, a esplenectomia com a ligadura das varizes esofagianas. Os resultados tardios deste tratamento (seguimento de 25 anos), publicados em 1982, demonstraram que, além de uma mortalidade operatória baixa, a recidiva de sangramento se manteve em índices semelhantes aos das cirurgias de derivação, com a vantagem da ausência de encefalopatia ${ }^{6}$.

Com o aperfeiçoamento das técnicas de escleroterapia, julgamos que a abordagem cirúrgica das varizes esofagianas poderia ser desnecessária e acrescentar um risco operatório ao procedimento. Com um estudo prospectivo e randomizado, comprovamos este pensamento ${ }^{7}$. Ou seja, a ligadura das varizes esofagianas intra-operatórias poderia ser substituída pela esclerose endoscópica pós-operatória.

Baseados ainda em dados anatômicos e fisiopatológicos do sistema porta e dos possíveis benefícios da ligadura da veia gástrica esquerda durante a esplenectomia, tanto nas varizes esofagianas, baixando sua pressão, quanto no fluxo da veia porta e arterialização do fígado, acrescentamos ao procedimento a ligadura da veia gástrica esquerda ${ }^{8}$.

Deste modo, desde 1992, realizamos, no Serviço de Cirurgia Geral do Hospital das Clínicas da Universidade Federal de Pernambuco, a esplenectomia + ligadura da veia gástrica esquerda (LVGE) + desvascularização da grande curvatura do estômago + esclerose endoscópica pós-ope- ratória no tratamento da hipertensão portal-esquistossomótica com antecedente de hemorragia digestiva. Quando da existência de varizes de fundo gástrico, realizamos ainda a abertura do fundo gástrico para a realização de uma sutura obliterante destas varizes.

O objetivo deste trabalho foi o de avaliar a recidiva de hemorragia digestiva alta, repercussões laboratoriais e mortalidade do tratamento cirúrgico da hipertensão portal esquistossomótica com antecedente de hemorragia digestiva, utilizando a esplenectomia + ligadura da veia gástrica esquerda (LVGE) + desvascularização da grande curvatura do estômago + esclerose endoscópica pós-operatória.

\section{MÉTODO}

No período de janeiro de 1992 a abril de 1998 foram realizados, no Serviço de Cirurgia Geral do Hospital das Clínicas da Universidade Federal de Pernambuco, 131 esplenectomias + ligadura da veia gástrica esquerda (LVGE) + desvascularização da grande curvatura do estômago + esclerose endoscópica pós-operatória para o tratamento da hipertensão portal esquistossomótica com antecedentes de hemorragia digestiva.

Quando da presença de varizes de fundo gástrico (41/111), foi associada ao procedimento cirúrgico a abertura do estômago e sutura das varizes de fundo gástrico. Durante o procedimento cirúrgico, foi realizada uma biópsia hepática em cunha de todos os pacientes.

Todos os pacientes foram solicitados a retornar ao ambulatório de Hipertensão portal e Fígado do Hospital das Clínicas para a realização de exames hematológicos e bioquímicos seguidos de endoscopia e ultra-sonografia e, ultra-sonografia Doppler do fígado, para avaliação do fluxo portal.

Dos 131 pacientes operados, 111 retornaram ao chamado ou foram identificados para seguimento. Em 11 pacientes o prontuário solicitado não coincidiu com o procedimento realizado ou foi extraviado, e nove pacientes não responderam ao chamado de comparecer ao Hospital. O seguimento médio foi de 30 meses.

Os 111 pacientes apresentavam epidemiologia positiva para a esquistossomose mansônica, caracterizada por banho de rio em área endêmica. Todos os pacientes apresentavam antecedentes de hemorragia digestiva exteriorizada por hematemese (99/111) ou por melena (12/111).

Os critérios de inclusão foram:

- idade superior a 16 anos;

- antecedente de hemorragia digestiva;

- varizes esofagianas ao exame endoscópico;

- hematócrito acima de 22;

- atividade enzimática não inferior a 50\%;

- exames de sorologia viral para hepatite negativos;

- confirmação da biópsia hepática de doença esquistossomótica pura.

Os critérios de exclusão foram:

- hepatopatia mista;

- passado de alcoolismo;

- trombose da veia porta. 
A idade dos pacientes variou entre 22 e 75 anos, com uma média de 45,1 anos. Cinqüenta e cinco pacientes eram do sexo masculino, e 56 do sexo feminino.

Com relação ao procedimento cirúrgico propriamente dito, a permanência hospitalar média foi de sete dias e em 38 pacientes houve a necessidade de transfusão sangüínea no peroperatório. Dois pacientes foram a óbito no pósoperatório imediato.

Os pacientes foram operados de maneira eletiva, fora do quadro agudo de hemorragia digestiva

A classificação do grau de fibrose esquistossomótica obedeceu aos critérios descritos por Coelho ${ }^{9}$. De acordo com a sua intensidade, o grau de fibrose foi classificado em:

- Grau I - os espaços portas apresentam-se com maior riqueza de células conjuntivas jovens, discreta produção de colágeno e presença variável de infiltrado inflamatório. A lâmina periportal e o retículo são normais.

- Grau II - há expansão do tecido conjuntivo com emissão de septos colágenos radiais, dando ao mesmo um aspecto estrelado.

- Grau III — os septos conjuntivos formam pontes com outros espaços portas ou com a veia, havendo neoformação angiomatóide bem evidente.

$\mathrm{Na}$ análise estatística dos dados, foi utilizado um teste de " $t$ " para diferença de médias, com nível de significância de $95 \%$.

\section{RESULTADOS}

O seguimento pós-operatório variou de um a 64 meses, com uma média de 30 meses.

O tratamento cirúrgico da hipertensão portal esquistossomótica através da esplenectomia + devascularização da grande curvatura do estômago + ligadura da veia gástrica esquerda + biópsia hepática apresentou, em um seguimento médio de 30 meses, recidiva hemorrágica de 14,4\% $(16 / 111)$ e mortalidade de 5,4\% (6/111). A recidiva de hemorragia digestiva alta foi exteriorizada através de hematemese em oito pacientes, todos por melena.

Dos seis pacientes, três foram a óbito com o diagnóstico de linfoma (não Hodgkin), hepatocarcinoma e infarto agudo do miocárdio, respectivamente. Dois pacientes foram a óbito no pós-operatório imediato, em conseqüência de sepse e de coagulação intravascular disseminada. $\mathrm{O}$ sexto paciente foi a óbito por recidiva da hemorragia digestiva alta. Além desses casos, dois pacientes apresentaram complicações graves decorrentes do procedimento cirúrgico. Um paciente apresentou dois episódios de acidente vascular cerebral isquêmico (AVCI) e o outro evoluiu com trombose mesentérica que necessitou tratamento cirúrgico e ressecção de cerca de $90 \mathrm{~cm}$ de alças de delgado.

A complementação do tratamento cirúrgico com escleroterapia endoscópica pós-operatória apresentou uma adesão de 32,4\% (36/111). Dos 36 pacientes que aderiram ao programa de escleroterapia endoscópica pós-operatória, em $19(52,7 \%)$ houve desaparecimento por completo das varizes esofágicas. Dos 75 pacientes que não realizaram escleroterapia endoscópica pós-operatória, 44 realizaram endoscopia digestiva alta, na ocasião do seguimento, e em apenas oito pacientes $(18,2 \%)$ foi identificada a ausência das varizes em decorrência do procedimento cirúrgico isolado.

A permanência hospitalar pós-operatória apresentou uma média de sete dias, variando de dois a 64 dias. Setenta e três pacientes $(65,7 \%)$ foram operados sem a utilização de hemoderivados.

No quadro 1 estão discriminados os dados relacionados à recidiva da hemorragia digestiva alta e do procedimento cirúrgico.

Quarenta e um pacientes $(36,9 \%)$ apresentavam no pré-operatório varizes de fundo gástrico. Nestes pacientes, o procedimento cirúrgico foi acrescido da abertura do estômago e ligadura das varizes de fundo gástrico. Este procedimento erradicou as varizes do fundo gástrico em 31 pacientes $(75,6 \%)$.

Em sete pacientes, as varizes de fundo gástrico surgiram após a operação. Em apenas um destes pacientes foi diagnosticada trombose da veia porta.

O grau de fibrose periportal das biópsias hepáticas operatórias variou de I a III, como esquematizado na Tabela 1. A recidiva hemorrágica foi estatisticamente maior no grupo de pacientes portadores de fibrose periportal graus II e III

\section{Quadro 1}

Resultados da Esplenectomia + devascularização da grande curvatura do estômago + ligadura da veia gástrica esquerda + escleroterpia endoscópica pós-operatória (Seguimento Médio $=30$ meses)

\begin{tabular}{|c|c|c|c|}
\hline & $\begin{array}{c}\text { № de } \\
\text { pacientes }\end{array}$ & $\begin{array}{l}N^{o} \text { de } \\
\text { casos }\end{array}$ & $\%$ \\
\hline Recidiva sangramento & \multirow[t]{3}{*}{111} & 16 & 14,4 \\
\hline Hematêmese & & 8 & 7,2 \\
\hline Melena & & 8 & 7,2 \\
\hline Mortalidade total & \multirow[t]{3}{*}{111} & 6 & 5,4 \\
\hline Operatória & & 2 & 1,8 \\
\hline Tardia & & 4 & 3,6 \\
\hline Permanência hospitalar & 111 & \multicolumn{2}{|c|}{ 7,0 dias (média) } \\
\hline Trombose da Veia Porta & 68 & 9 & 13,2 \\
\hline Escleroterapia pós-operatória & 111 & 36 & 32,1 \\
\hline Erradicou varizes do esôfago & 36 & 19 & 52,7 \\
\hline \multicolumn{4}{|l|}{ Cirurgia sem escleroterapia } \\
\hline pós-operatório & 75 & 44 & 58,6 \\
\hline Erradicou varizes & 44 & 8 & 18,2 \\
\hline Varizes de fundo gástrico & 111 & 41 & 36,9 \\
\hline \multicolumn{4}{|l|}{ Calibre da veia Porta } \\
\hline Pré-operatório & 50 & 1,32 & $\mathrm{p}<0,001$ \\
\hline Pós-operatório & 68 & 0,92 & \\
\hline
\end{tabular}


quando comparados com o grupo de grau I. Não houve relação entre o grau de fibrose periportal e a presença de varizes de fundo gástrico e o calibre da veia porta.

Os resultados das análises hematológicas e bioquímicas pré e pós-operatórias estão dispostas na Tabela 2.

Apesar de ter sido solicitada ultra-sonografia com Doppler para todos os 111 pacientes, apenas 68 pacientes $(70,2 \%)$ realizaram o exame com análise do fluxo portal. Em nove pacientes $(13,2 \%)$, foi diagnosticada trombose da veia porta; em dois pacientes foi diagnosticada a presença de trombo na veia porta e em um paciente foi diagnosticada trombose da veia mesentérica superior.

Dos nove pacientes que apresentavam trombose da veia porta, um paciente foi a óbito por recidiva de hemorragia digestiva dois meses após a operação. Em outros dois pacientes, houve recidiva de hemorragia digestiva com presença de varizes esofagianas de grosso calibre. Os outros seis pacientes encontram-se assintomáticos, sendo a trombose portal um achado da ultra-sonografia Doppler de controle. Destes seis pacientes, cinco apresentam varizes de esôfago.

\section{DISCUSSÃO}

A cirurgia da hipertensão portal esquistossomótica difere essencialmente da cirurgia da hipertensão portal de pacientes cirróticos pelo fato de que a doença esquistossomótica preserva, de certa maneira, a função hepática. Deste modo, na tentativa de se reduzir a pressão portal, quer pelo hiperfluxo, quer pelo bloqueio pré-sinusoidal, o cirurgião deve sempre ter em mente que a operação poderá interferir na perfusão hepática e, conseqüentemente, na funcionalidade do fígado ${ }^{10}$.

As duas correntes do tratamento cirúrgico da hipertensão portal esquistossomótica advogam a operação de derivação de maneira seletiva (espleno-renal distal) ou as operações de desconexões associadas à esplenectomia.

Tabela 1

Grau de fibrose periportal das biópsias hepáticas e sua relação com a recidiva hemorrágica, presença pré-operatória de varizes de fundo gástrico e calibre pré-operatório da veia porta

\begin{tabular}{l|c|c|c|c}
\hline Fibrose periportal & $N^{o}$ & Recidiva hemorrágica & Varizes de fundo gástrico & Calibre da vv. porta \\
\hline Grau I & 29 & $1-3,4 \%$ & $8-27,5 \%$ & $1,35 \mathrm{~cm}$ \\
Grau II & 38 & $8-21,0 \% *$ & $16-42,1 \%$ & $1,37 \mathrm{~cm}$ \\
Grau III & 44 & $7-15,9 \% *$ & $17-40,9 \%$ & $1,37 \mathrm{~cm}$ \\
\hline Total & 111 & $16-14,4 \%$ & $41-36,9 \%$ &
\end{tabular}

$* p<0,05$ em relação ao Grau I.

Tabela 2

Discriminação dos dados hematológicos e bioquímicos pré e pós-operatórios

\begin{tabular}{lc|c|c}
\hline & Pré-operatório & Pós-operatório & $p$ \\
\hline Hematócrito & 31,60 & 39,17 & $<0,001$ \\
Hemoglobina & 10,21 & 13,00 & $<0,001$ \\
Leucócitos & 4162,18 & 7037,31 & $<, 001$ \\
Linfócitos & 824,30 & $2.151,19$ & $<001$ \\
Plaquetas & $115.621,20$ & $297.452,60$ & $<001$ \\
Tempo de protombina & 14,91 & 12,66 & $<001$ \\
Glicose & 98,73 & 101,95 & $<0,05$ \\
Uréia & 29,65 & 28,23 & $<0,001$ \\
Creatinina & 0,83 & 0,73 & $<0,001$ \\
Albumina & 3,58 & 3,97 & \\
TGO & 37,05 & 51,38 & \\
TGP & 32,30 & 43,21 & \\
Bilirrubinas totais & 0,93 & 0,93 & 0,30 \\
Bilirrubina direta & 0,36 & 2 & \\
\hline
\end{tabular}


Os resultados quanto à recidiva da hemorragia a curto e a longo prazo se eqüivalem, mas, tendo uma experiência de mais de 40 anos com o tratamento da hipertensão portal esquistossomótica utilizando a esplenectomia associada à atuação direta nas varizes esofagianas, verificando resultados plenamente satisfatórios, continuamos a utilizar este tipo de tratamento no Serviço de Cirurgia Geral do HC da UFPE. Vale ressaltar que, ao longo dos anos, vem-se tentando associar novos procedimentos, com o intuito de melhorar ainda mais os resultados 6 .

Este trabalho visa essencialmente à revisão de uma mudança de conduta em nosso hospital com base em experiências anteriores do próprio serviço.

Kelner et al $^{6}$ publicaram, em 1982, uma experiência com seguimento de 25 anos de pacientes esquistossomóticos tratados cirurgicamente com esplenectomia e ligadura das varizes esofagianas. A recidiva hemorrágica foi de $12,6 \%$ e uma mortalidade tardia de 20,38\%, por motivos não completamente esclarecidos. Com o advento da escleroterapia e sua utilização no tratamento de varizes esofagianas, com resultados bastante satisfatórios, desenhamos um estudo comparando três tipos de tratamento: escleroterapia isolada, escleroterapia + esplenectomia e a esplenectomia com ligadura de varizes esofagianas. A recidiva hemorrágica do grupo de escleroterapia isolada foi estatisticamente maior do que os outros dois grupos. Os resultados do grupo de escleroterapia + esplenectomia e do da esplenectomia + ligadura de varizes esofagianas foram semelhantes. Desta maneira, por apresentar uma morbidade menor, deixamos de realizar a ligadura das varizes esofagianas durante a cirurgia e passamos a adotar a escleroterapia pós-operatória ${ }^{7}$.

Outra modificação importante no tratamento cirúrgico dos pacientes esquistossomóticos foi a realização, de rotina, da ligadura da veia gástrica esquerda associada a uma desvascularização da grande curvatura do estômago. A ligadura da veia gástrica esquerda visa a diminuir a pressão nas varizes esofagianas e, por outro lado, a diminuir o fluxo hepatofugal, reduzindo a incidência de trombose da veia porta e, conseqüentemente, preservando a função hepática ${ }^{8}$.

Na casuística apresentada, o índice de recidiva hemorrágica, em um seguimento de 30 meses, foi de 14,4\%, com uma mortalidade de 5,4\% (6/111). Dos seis pacientes que foram a óbito, três apresentavam diagnóstico de linfoma, hepatocarcinoma e infarto agudo do miocárdio, respectivamente. Dois pacientes foram a óbito no pósoperatório imediato, em conseqüência de sepse e de coagulação intravascular disseminada, e apenas um paciente foi a óbito decorrente da recidiva de hemorragia digestiva alta. Talvez por apresentar um período de seguimento menor, quando comparado com os dados de Kelner et $\mathrm{al}^{6}$, conseguimos identificar a causa mortis dos seis pacientes que foram a óbito.
Apesar da orientação de se completar o tratamento cirúrgico pós-operatório com escleroterapia endoscópica, a adesão ao tratamento foi de apenas $32,1 \%$. Notamos que os pacientes que retornaram para a complementação endoscópica foram aqueles que no período de pós-operatório apresentaram recidiva da hemorragia digestiva. No entanto, a complementação com escleroterapia pós-operatória determinou uma erradicação das varizes esofagianas em torno de $52,7 \%$. Nos pacientes que não realizaram a escleroterapia pós-operatória, apenas $18,2 \%$ tiveram as varizes esofagianas erradicadas. Ou seja, o procedimento cirúrgico isolado (esplenectomia + devascularização da grande curvatura do estômago + ligadura da veia gástrica esquerda) erradicou apenas $18,2 \%$ das varizes esofagianas. Desta forma, recomendamos a complementação cirúrgica com escleroterapia endoscópica pós-operatória.

As varizes de fundo gástrico representam, ao nosso ver, um problema à parte. Quando diagnosticadas pré-operatoriamente, as abordamos diretamente durante o procedimento, através da abertura do fundo gástrico e sutura direta destas varizes. Este procedimento erradicou 75,6\% destas varizes. No entanto, em sete pacientes o desenvolvimento das varizes de fundo gástrico se deu após a cirurgia, e em apenas um, identificamos uma causa fisiopatológica para o caso (trombose da veia porta).

A abordagem direta das varizes de fundo gástrico é fundamental, pois seu tratamento por via endoscópica é de difícil execução, e geralmente é acompanhado de quadros graves de hemorragia digestiva alta.

Em nossa casuística, identificamos, com a realização da ultra-sonografia com Doppler, 13,2\% de casos com trombose da veia porta. Este número é bem abaixo dos $30 \%$ da desconexão ázigo-portal ${ }^{11}$, e bem próximo dos $15,8 \%$ determinados pela esplenectomia e ligadura das varizes do esôfago ${ }^{8}$.

O grau de fibrose hepática tem sido correlacionado com o diâmetro das veias porta e esplênica e os diâmetros longitudinal, transverso e ântero-posterior do baço ${ }^{12}$. Não identificamos relação entre o grau de fibrose hepática e a presença de varizes de fundo gástrico ou com o calibre da veia porta. Os pacientes que apresentavam fibrose periportal grau II ou III apresentaram um índice de recidiva hemorrágica estatisticamente maior do que os que apresentavam grau I de fibrose.

$\mathrm{Na}$ visão dos autores, o tratamento cirúrgico da hipertensão esquistossómotica proposto neste trabalho resultou em recidivas similares aos da literatura o que, principalmente, é um procedimento relativamente simples, com baixas taxas de morbimortalidade operatórias, não comprometendo a funcionalidade hepática no pósoperatório. A utilização da ligadura da veia gástrica esquerda e a abordagem direta das varizes de fundo gástrico determinaram ainda uma atuação mais efetiva nas varizes esofagogástricas e, talvez, um menor percentual de casos de trombose da veia porta. 


\begin{abstract}
Background: At the Clinical Hospital of the Federal University of Pernambuco the surgical treatment of hepatosplenic shistosomiasis has been done with splenectomy + left gastric vein ligature $(L G V L)+$ devascularization of the great curvature of the stomach + postoperative endoscopic sclerosis. If the patient has gastric fundus variceals, the gastric fundus was open and the variceals sutured. The objective of this paper was to evaluate the surgical treatment proposal regarding the re-bleeding rate, mortality and laboratorials changes. Method: During the period between 1992 and April 1998, 131 procedures in the General Surgery Division of the Clinical Hospital. The patients were asked to return to the Hospital and underwent a clinical/laboratorial analysis. The mean follow-up was 30 months. Results: The re-bleeding rate was $14,4 \%$ (16/111) and the mortality rate 5,4\% (6/111). In 8 cases of re-bleeding the exteriorization was in form of melena and in 8 as hematemesis. In 3 cases the mortality was resulted of a linfoma, a hepatocarcinoma and a cardiac stroke. In two patients the death was resulted from the immediate postoperative period (sepsis and intravascular disseminated coagulation). The other death was during the late postoperative period as a result of a re-bleeding episode. Nine patients $(13,2 \%)$ evaluated with portal vein thrombosis and in two a superior mesenteric vein thrombosis was identified. Hematological and biochemical data's was also analyzed. Conclusions: The authors concluded that the surgical treatment of the hepatosplenic shistosomiasis with splenectomy $+L G V L+$ devascularization of the great curvature of the stomach + postoperative endoscopic sclerosis is a safe procedure and with results comparable with the literature, and a advantage to maintain the liver functionality. The LGVL and the direct suture of the gastric fundus variceals gave a more effective action on the esophagus-gastric variceals and a possible lower rates of portal vein thrombosis.
\end{abstract}

Key Words: Hepatosplenic shistosomiasis; Splenectomy; Portal vein thrombosis

\section{REFERÊNCIAS}

1. Magalhães Filho A, Silva JF. Patologia e Patogenia da Esquistossomose mansônica. In: Malta J. Esquistossomose Mansônica. Recife, Editora da UFPE: 61-77, 1994.

2. Coutinho AD.Terapêutica etiológica da esquistossomose mansônica e sua avaliação. In. Malta J. Esquistossomose Mansônica. Recife, Editora da UFPE: 203-214, 1994.

3. Kelner S, Silveira M. História natural das varizes do esôfago na esquistossomose mansônica hepatoesplênica. In: Kelner S, Silveira M. Varizes do esôfago na esquistossomose mansônica. Recife, Editora da UFPE: 55-61, 1997.

4. Kelner S, Ferraz EM, Wanderley F. Hematêmese: inquérito sobre desencadeamento por drogas contendo ácido acetilsalicílico na hipertensão porta esquistossomótica. Fac Med Univ Recife, 1964, 24:153-165.

5. Kelner S. Avaliação da esplenectomia e ligadura intraeosafiana das varizes do esôfago na esquistossomose mansônica. Recife, 1965. Tese para Professor Catedrático da Faculdade de Medicina da Universidade do Recife.

6. Kelner S, Ferreira PR, Dantas A et al. Ligadura de varizes esôfago-gástricas na hipertensão porta esquistossomótica: evolução de 25 anos. Rev Col Bras Cir, 1982, 9:140-146.

7. Souza Jr EC, Leôncio MP, Ferraz EM. Tratamento cirúrgico da hipertensão porta esquistossomótica: Estudo prospectivo randomizado de três modalidades terapêuticas. Rev Col Bras Cir, 1977, 24:98.

8. Lacerda CM. Alterações angiográficas e pressóricas determinadas pela esplenectomia e ligadura interna de varizes de esôfago na esquistossomose mansônica. 1991 São Paulo, Tese Doutorado - Faculdade de Medicina da Universidade de São Paulo.

9. Coelho RB. Lesões hepáticas secundárias. In: Coelho RB. Anatomia patológica das afecções hepáticas. Recife, Editora da UFPE: 59-77, 1971.

10. Ferraz EM, Ferraz AAB. Tratamento cirúrgico da hipertensão portal esquistossomótica. In: Malta J. Esquistossomose Mansônica. Recife, Editora da UFPE: 235249, 1994

11. Carneiro JL, Tabachi JR. Controvérsias sobre a cirurgia de eleição na hipertensão portal. In: Abrantes W. Hipertensão portal - estado atual. Clínica Brasileira de Cirurgia Colégio Brasileiro de Cirurgiões, 1995, 2(1):137170.

12. Domingues ALC. Ultra-sonografia na esquistossomose mansônica hepato-esplênica: avaliação da intensidade da fibrose periportal e da hipertensão porta. Tese de Doutorado em Medicina - Universidade Federal de Pernambuco, 1998.

Endereço para correspondência

Dr. Álvaro Antônio B. Ferraz

Av. Beira Rio, 240/2.501

50750-400_Recife-PE

E-mail:aabf@truenet.com.br 